\title{
9-Arylvinyl- and 9,10-bis(arylvinyl)-anthracenes with phenyl and naphthyl as aryl moieties: Differential aggregation behaviors and optoelectronic properties
}

\author{
Jing Dong ${ }^{\mathrm{a}}$, Wei Liu ${ }^{\mathrm{a}}$, Shian Ying ${ }^{\mathrm{a}}$, Yongjin $\mathrm{Wu}^{\mathrm{a}}$, Shanfeng Xue ${ }^{\mathrm{a}, \mathrm{b}, *}$, Wenjun Yang ${ }^{\mathrm{a}} *$ \\ ${ }^{a}$ Key Laboratory of Rubber-Plastics of Ministry of Education/Shandong \\ Province (QUST), School of Polymer Science \& Engineering, Qingdao \\ University of Science \& Technology, 53-Zhengzhou Road, Qingdao 266042, \\ China. \\ Tel: +86 532 84023670; fax: +86 53284023977 . \\ E-mail address: ywjph2004@qust.edu.cn; sfxue@qust.edu.cn \\ ${ }^{\mathrm{b}}$ State Key Laboratory of Luminescent Materials and Devices, Institute of \\ Polymer Optoelectronic Materials and Devices, South China University of \\ Technology, Guangzhou 510640, P. R. China.
}

\begin{abstract}
In this paper, we have synthesized four simple twisting arylvinyl anthracene derivatives, 9-styrylanthracene (ASB), 9,10-bis(styryl)anthracene (SB), 9-(2-(naphthalen-1-yl)vinyl)anthracene (ASN) and 9,10-bis((naphthalen-1-yl)vinyl)anthracene (SN), to demonstrate the effect of skeleton symmetry on optical and optoelectronic properties. The results showed that $\mathbf{S B}$ and $\mathbf{S N}$ are weak emission with the fluorescence quantum yields $(\Phi)$ of ca. $7 \%$ but ASB and ASN are highly fluorescent $(\Phi \approx 50 \%)$ in THF solution, although they all exhibit high solid-state fluorescence efficiencies (22 50\%). Grinding could induce about $20 \mathrm{~nm}$ of spectral shift of ASB and ASN crystalline fluorescence, but SB and SN crystals hardly exhibit mechanofluorochromic behavior. Furthermore, the evaporated films of SB and $\mathbf{S N}$ are fragile due to re-crystallization soon, but those of ASB and ASN are stable and could be used as the bulk emissive layer exhibiting commendable electroluminescent performances. This finding indicates that the geometric structures of conjugated skeletons could influence the electronic and aggregate structure of organic luminogens, and then alter their optical and optoelectronic properties.
\end{abstract}

Keywords: 9-Arylanthracene; 9,10-Bis(aryl)anthracene; Geometric effect; Electroluminescence; Piezofluorochromism.

\section{Introduction}


Conjugated organic molecules exhibiting high solution and solid-state fluorescence efficiency are promising materials for the potential applications in fluorescence imaging, chemo-sensing, light emitting diodes, and active gain media for optically pumped solid-state lasers [1-12]. However, common organic chromophores show aggregation- or crystallization-quenched emission (AQE) effect due to strong intermolecular $\pi-\pi$ stacking interactions, a typical problem of most fluorescent organic molecules [13,14]. To alleviate or overcome this problem, both the introduction of bulky sterical hindrance groups and the construction of twisted skeleton conformation are effective approaches $[15,16]$. Since Tang's discovery, conjugated organic molecules with twisted skeletons bearing rotatable aryl moieties have attracted much attention due to their aggregation-induced emission (AIE) or crystallization-enhanced emission (CEE) effects [17-24]. The emergence of these new $\pi$-conjugated organic materials has widened the scope of molecular candidates applicable in optics and optoelectronics. 9,10-Bis(arylvinyl)anthracene derivatives are just a class of promising and attractive AIE/CEE molecules, and their AIE behavior was first demonstrated by Prasad et al. in 9,10-bis[4'-(4"-aminostyryl)styryl]anthracene and used in two-photon fluorescence imaging and photodynamic therapy [25-27]. Recently, there have been a variety of reports on the synthesis and applications of 9,10-bis(arylvinyl)anthracenes[27]. It has been found that most 9,10-divinylanthracene derivatives are not only AIE/CEE active but also piezofluorochromic. 9-Arylvinylanthracenes are also skeleton-twisted dyes; however, their aggregation behavior and optoelectronic properties are rarely investigated. We considered that the geometric symmetry of conjugated skeletons could influence the electronic and aggregate structure of organic luminogens, thus their optical and optoelectronic properties might be tuned or changed by changing substitution patterns. Here we have synthesized four simple anthrylvinyl derivatives, 9-styrylanthracene (ASB), 9-(2-(naphthalen-1yl)vinyl-2)anthracene (ASN), 9,10-bis(styryl)anthracene (SB), and 9,10-bis((naphthalen-1-yl)vinyl-2)anthracene (SN), to investigate the effect of skeleton symmetry on the optical and optoelectronic properties. We now report that 9-arylvinyl- and 9,10-bis(arylvinyl)-anthracenes exhibit differential optical and optoelectronic properties.

\section{Experimental}

\subsection{Materials}

Solvents and reagents were all commercially available analytical-grade products and used as received without further purification, unless otherwise stated. Tetrahydrofuran (THF) was distilled over metallic sodium and dimethyl formamide (DMF) over calcium hydride before use. Anthracene, benzaldehyde, 1-naphthaldehyde, charge-transporting materials, and catalysts were purchased 
from Aldrich Chemical Co. or Energy Chemical Co., China. 9,10-Bis(diethoxylphosphorylmethyl)anthracene is from previous work [28].

\subsection{Measurements}

${ }^{1} \mathrm{H}$ and ${ }^{13} \mathrm{C}$ NMR spectra were recorded on a Bruker AC500 (500/125 MHz) spectrometer using deuterated chloroform $\left(\mathrm{CDCl}_{3}\right)$ as solvents and tetramethylsilane (TMS) as internal standard. UV-vis absorption spectra were recorded on a Hitachi U-4100 spectrophotometer. Fluorescence measurements were carried out with a Hitachi F-4600 spectrophotometer. Solution fluorescence quantum yield $(\Phi)$ was determined at room temperature by the relative dilution method using quinine (in $0.1 \mathrm{M} \mathrm{H}_{2} \mathrm{SO}_{4}$ ) as the reference [28]. Solid-state fluorescence efficiencies were measured on an integrating sphere (C-701, Labsphere) with a $325 \mathrm{~nm}$ Xe light as the excitation source. Cyclic voltammetry (CV) was performed with a BAS 100W Bioanalytical Systems, using a glass carbon disk (diameter $=3 \mathrm{~mm}$ ) as the working electrode, a platinum wire as the auxiliary electrode with a porous ceramic wick, and $\mathrm{Ag} / \mathrm{Ag}^{+}$as the reference electrode standardized by the redox couple ferrocenium/ferrocene. All solutions were purged with a nitrogen stream for $10 \mathrm{~min}$ before measurement. The procedure was performed at room temperature, and a nitrogen atmosphere was maintained over the solution during measurements. Differential scanning calorimetry (DSC) was performed on a Netzsch DSC204F1 at a heating rate of $10{ }^{\circ} \mathrm{C} \mathrm{min}{ }^{-1}$.

\subsection{Device Fabrication}

Indium-tin oxide (ITO) coated glass with a sheet resistance of $15-20 \Omega / \mathrm{cm}^{2}$ was used as the cathode whose substrate was pre-patterned by photolithography to reserve an effective device size of $6.25 \mathrm{~mm}^{2}$. Then, it was cleaned in an ultrasonic bath with acetone, detergent, deionized water, and isopropanol as the clean agent. After being dried in an oven, it is treated with oxygen plasma for 4 min, and then $40 \mathrm{~nm}$ of PEDOT:PSS layer was spin-coated and baked overnight in a vacuum oven at $80{ }^{\circ} \mathrm{C}$. On the top of above active layer, N,N'-di(1-naphthyl)-N,N'-diphenyl-(1,1'-biphenyl)-4,4'-diamine (NPB) as holetransporting layer (40 nm), ASB (or ASN) as bulk emitting layer (EML, $20 \mathrm{~nm}$ ) and 1,3,5-tri(phenyl-2-benzimidazolyl)benzene (TPBi) as electron-transporting layer $(50 \mathrm{~nm})$ were vacuum deposited in a sequence. Finally, $0.5 \mathrm{~nm}$ of $\mathrm{LiF}$ film and $100 \mathrm{~nm}$ of $\mathrm{Al}$ film were vacuum deposited to form the anode at a base pressure of $3 \times 10^{-4} \mathrm{~Pa}$. The thickness of each deposition layer was monitored using a quartz crystal thickness/ratio monitor (STM-100/MF, Sycon). Electroluminescence (EL) spectra were measured by a PR650 fluorescence spectrophotometer. Luminance-voltage and current density-voltage characteristics were recorded simultaneously by combining the spectrometer with a Keithley model 2400 programmable voltage-current source. All measurements were carried out at room temperature under ambient conditions.

\subsection{Synthesis}




\subsubsection{Synthesis of 9-anthraldehyde (1)}

A mixture of anthracene $(6.0 \mathrm{~g}, 35.2 \mathrm{mmol})$ and phosphorus oxychloride (4 $\mathrm{mL}, 72 \mathrm{mmol}$ ) was heated for $1 \mathrm{~h}$ at $90{ }^{\circ} \mathrm{C}$, and DMF (4 mL, $50.6 \mathrm{mmol}$ ) was added dropwise over $30 \mathrm{~min}$. The resulting mixture was stirred at $95{ }^{\circ} \mathrm{C}$ for 15 $\mathrm{h}$ and then cooled to $55^{\circ} \mathrm{C}$. After $40 \mathrm{~mL}$ of hot water $\left(60{ }^{\circ} \mathrm{C}\right)$ was added, the mixture was warmed to $65{ }^{\circ} \mathrm{C}$ and stirred for $30 \mathrm{~min}$. The $\mathrm{pH}$ was adjusted to between 4 and 6 by slowly adding aqueous $\mathrm{NaOH}$ under vigorous stirring. The mixture was cooled to $50{ }^{\circ} \mathrm{C}$ and laid up for $30 \mathrm{~min}$ without stirring. After cooled to room temperature, the filtrate was evaporated under reduced pressure. The resulting dark yellow solid was re-crystallized from acetic acid to give yellow crystals $\left(6.0 \mathrm{~g}, 82.5 \%\right.$ yield). ${ }^{1} \mathrm{H} \mathrm{NMR}\left(500 \mathrm{MHZ}, \mathrm{CDCl}_{3}\right): \delta(\mathrm{ppm}) 7.56$ $(\mathrm{t}, J=10 \mathrm{~Hz}, 2 \mathrm{H}), 7.70(\mathrm{t}, J=7.5 \mathrm{~Hz}, 2 \mathrm{H}), 8.09$ (d, $J=7.5 \mathrm{~Hz}, 2 \mathrm{H}), 8.72$ (s, 1H), $9.01(\mathrm{~d}, J=10 \mathrm{~Hz}, 2 \mathrm{H}), 11.55(\mathrm{~s}, 1 \mathrm{H})$.

\subsubsection{Synthesis of 9-anthracenemethanol (2)}

9-Anthraldehyde $(2.0 \mathrm{~g}, 9.6 \mathrm{mmol})$ in absolute ethanol $(10 \mathrm{~mL})$ was added slowly a mixture of sodium borohydride $(1.0 \mathrm{~g}, 26.4 \mathrm{mmol}$, in $10 \mathrm{~mL}$ of ethanol) at room temperature. After stirring for $2 \mathrm{~h}$, water $(5 \mathrm{~mL})$ was poured into the mixture. After filtration, light yellow solid was obtained. This compound was used for next step without further purification.

\subsubsection{Synthesis of 9-bromomethyl anthracene (3)}

A cold solution $\left(0{ }^{\circ} \mathrm{C}\right)$ of 9-Anthracenemethanol $(1.5 \mathrm{~g}, 27.2 \mathrm{mmol})$ in toluene $(45 \mathrm{~mL})$ was slowly added $\mathrm{PBr}_{3}(0.4 \mathrm{~mL}, 4.1 \mathrm{mmol})$. The reaction mixture was stirred for $1 \mathrm{~h}$ at $0{ }^{\circ} \mathrm{C}$ and then stirred for $1 \mathrm{~h}$ at room temperature. The mixture was adjusted to $\mathrm{pH}=7$ by adding saturated sodium carbonate solution. The organic phase was separated and the aqueous phase was washed three times with toluene, and combined organic phase was dried over $\mathrm{MgSO}_{4}$ and concentrated under reduced pressure. The crude product was re-crystallized from toluene in refrigerator $\left(-10^{\circ} \mathrm{C}\right)$ to afford yellow crystals $(1.15 \mathrm{~g}, 59.2 \%)$. This compound was used for next step without further purification.

\subsubsection{Synthesis of 9-diethoxylphosphorylmethylanthracene (4)}

The mixture of 9-bromomethyl anthracene $(5.0 \mathrm{~g}, 18.5 \mathrm{mmol})$ and triethylphosphite $(10 \mathrm{~mL})$ was gently refluxed overnight. After cooling, the excess triethylphosphite was removed under reduced pressure. The crude product was separated by silica gel column chromatography using ethyl acetate/petroleum ether (8/1) as the eluent. A yellow solid was obtained (1.0 g. 74.3\% yield). ${ }^{1} \mathrm{H}$ NMR (500 MHZ, $\left.\mathrm{CDCl}_{3}\right): \delta(\mathrm{ppm}) 1.07(\mathrm{t}, J=5.00 \mathrm{~Hz}, 6 \mathrm{H})$, $3.80(\mathrm{q}, 4 \mathrm{H}), 4.21(\mathrm{~s}, 1 \mathrm{H}), 4.25(\mathrm{~s}, 1 \mathrm{H}), 7.47(\mathrm{t}, J=6.50 \mathrm{~Hz}, 2 \mathrm{H}), 7.56(\mathrm{t}, J=$ $6.50 \mathrm{~Hz}, 2 \mathrm{H}), 8.00(\mathrm{~d}, J=10.00 \mathrm{~Hz}, 2 \mathrm{H}), 8.33$ (d, $J=10.00 \mathrm{~Hz}, 2 \mathrm{H}), 8.41$ (s, $1 \mathrm{H})$. 


\subsubsection{Synthesis of 9-styrylanthracene (ASB).}

The solution of 9-diethylphosphorylmethyl- anthracene $(0.50 \mathrm{~g}, 1.52 \mathrm{mmol})$ and benzaldehyde $(0.21 \mathrm{~g}, 1.98 \mathrm{mmol})$ in anhydrous THF $(20 \mathrm{~mL})$ was added potassium tert-butoxide $(1.37 \mathrm{~g}, 12.2 \mathrm{mmol})$ and stirred at room temperature for $8 \mathrm{~h}$. The mixture was added into methanol and the precipitate was collected. The crude product was separated by silica gel column chromatography using petroleum ether as the eluent $(0.34 \mathrm{~g}, 80 \%$ yield $) .{ }^{1} \mathrm{H}$ NMR $\left(500 \mathrm{MHZ}, \mathrm{CDCl}_{3}\right)$ : $\delta(\mathrm{ppm}) 6.96(\mathrm{~d}, J=16.60 \mathrm{~Hz}, 2 \mathrm{H}), 7.13(\mathrm{~m}, 1 \mathrm{H}), 7.36(\mathrm{~m}, 6 \mathrm{H}), 7.69(\mathrm{~d}, J=$ $7.70 \mathrm{~Hz}, 2 \mathrm{H}), 7.93(\mathrm{~d}, J=16.60 \mathrm{~Hz}, 1 \mathrm{H}), 8.02(\mathrm{~d}, J=8.55 \mathrm{~Hz}, 2 \mathrm{H}), 8.36(\mathrm{~d}, J=$ $8.75 \mathrm{~Hz}, 2 \mathrm{H}), 8.41(\mathrm{~s}, 1 \mathrm{H}) ;{ }^{13} \mathrm{C} \mathrm{NMR}\left(125 \mathrm{MHz}, \mathrm{CDCl}_{3}\right): \delta(\mathrm{ppm})$ 122.33, $124.84,125.14,125.43,125.99,126.44,126.56,127.98,128.66,128.80,129.69$, 131.46, 132.72, 137.26. Anal. Calcd for $\mathrm{C}_{22} \mathrm{H}_{16}$ : C, 94.25; H, 5.75. Found: C, $94.11 ; \mathrm{H}, 5.79$.

\subsubsection{Synthesis of 9-(2-(naphthalen-1-yl)vinyl)anthracene (ASN).}

This compound was synthesized by same procedure for ASB except that naphthaldehyde $(0.308 \mathrm{~g}, 1.97 \mathrm{mmol})$ was used. $0.41 \mathrm{~g}$ of product was obtained (82.3\% yield). ${ }^{1} \mathrm{H}$ NMR $\left(500 \mathrm{MHz}, \mathrm{CDCl}_{3}\right): \delta(\mathrm{ppm}) 7.51(\mathrm{~m}, 6 \mathrm{H}), 7.62(\mathrm{t}, J=$ $7.65 \mathrm{~Hz}, 1 \mathrm{H}), 7.76(\mathrm{~d}, J=16.25 \mathrm{~Hz}, 1 \mathrm{H}), 7.91(\mathrm{t}, J=8.55 \mathrm{~Hz}, 2 \mathrm{H}), 7.98(\mathrm{~d}, J=$ $16.20 \mathrm{~Hz}, 1 \mathrm{H}), 8.06(\mathrm{~m}, 3 \mathrm{H}), 8.18(\mathrm{~d}, J=8.40 \mathrm{~Hz}, 1 \mathrm{H}), 8.46(\mathrm{~S}, 2 \mathrm{H}), 8.48(\mathrm{~S}$, $1 \mathrm{H}) ;{ }^{13} \mathrm{C}$ NMR $\left(125 \mathrm{MHz}, \mathrm{CDCl}_{3}\right): \delta(\mathrm{ppm}) 122.37,123.79,125.20,125.59$, 125.77, 125.98, 126.29, 126.59, 127.86, 128.38, 128.71, 129.79, 131.33, 131.50, 132.95, 133.75, 134.78, 135.06. Anal. Calcd for $\mathrm{C}_{26} \mathrm{H}_{18}$ : C, 94.51; H, 5.49. Found: $\mathrm{C}_{26} \mathrm{H}_{18}: \mathrm{C}, 94.39 ; \mathrm{H}, 5.45$.

\subsubsection{Synthesis of 9,10-bis(styryl)anthracene (SB)}

9,10-Bis(diethylphosphorylmethyl)- anthracene (0.30 g, $0.627 \mathrm{mmol})$ and benzaldehyde $(0.15 \mathrm{~g}, 1.37 \mathrm{mmol})$ were dissolved in anhydrous THF $(20 \mathrm{~mL})$. Potassium tert-butoxide $(1.12 \mathrm{~g}, 9.98 \mathrm{mmol})$ was added and the resulting mixture was stirred at room temperature for $8 \mathrm{~h}$. Methanol was added into the mixture and the precipitate was collected. The crude product was separated by silica gel column chromatography using petroleum ether/dichloro-methane (4/1) as the eluent $\left(0.20 \mathrm{~g}, 83 \%\right.$ yield). ${ }^{1} \mathrm{H} \mathrm{NMR}\left(500 \mathrm{MHz}, \mathrm{CDCl}_{3}\right): \delta(\mathrm{ppm})$ $6.95(\mathrm{~d}, J=16.55 \mathrm{~Hz}, 2 \mathrm{H}), 7.37(\mathrm{t}, J=7.35 \mathrm{~Hz}, 2 \mathrm{H}), 7.47(\mathrm{~m}, 8 \mathrm{H}), 7.70(\mathrm{~d}, J=$ $7.65 \mathrm{~Hz}, 4 \mathrm{H}), 7.94(\mathrm{~d}, J=16.55 \mathrm{~Hz}, 2 \mathrm{H}), 8.40(\mathrm{~m}, 4 \mathrm{H})$. Anal. Calcd for $\mathrm{C}_{30} \mathrm{H}_{22}$ : C, 94.20; H, 5.80. Found: C, 94.28; H, 5.75.

\subsubsection{Synthesis of 9,10-bis((naphthalen-1-yl)vinyl-2)anthracene (SN)}

This compound was prepared by same procedure for SB except that naphthaldehyde $(0.308 \mathrm{~g}, 1.97 \mathrm{mmol})$ was used. The product was obtained by re-crystallization from chloroform $\left(0.38 \mathrm{~g}, 75 \%\right.$ yield). ${ }^{1} \mathrm{H}$ NMR $(500 \mathrm{MHz}$, $\left.\mathrm{CDCl}_{3}\right): \delta(\mathrm{ppm}) 7.54(\mathrm{~m}, 8 \mathrm{H}), 7.65(\mathrm{t}, J=7.65 \mathrm{~Hz}, 2 \mathrm{H}), 7.78(\mathrm{~d}, J=16.15 \mathrm{~Hz}$, 2H), $7.93(\mathrm{~m}, 4 \mathrm{H}), 8.02(\mathrm{~d}, J=16.15 \mathrm{~Hz}, 2 \mathrm{H}), 8.12(\mathrm{~d}, J=7.15 \mathrm{~Hz}, 2 \mathrm{H}), 8.20(\mathrm{~d}$, 


\section{Results and discussion}

\subsection{Synthesis and characterization}

The synthetic routes to four simple anthracene-based luminogens, ASB, ASN, SB, and SN, are depicted in Scheme 1. Briefly, the Wittig-Horner reaction between arylaldehydes and 9-diethylphosphorylmethyl- or 9,10-bis(diethoxyl- phosphorylmethyl)-anthracenes was employed, and the target products were characterized by NMR and elemental analysis. It should be noticed that, although the products could be obtained by Heck coupling of arylbromide and arylethene, the present reaction is versatile way due to no use of palladium catalysts, high yields, and easy operation. Moreover, the present 9-diethoxyl- phosphorylmethylanthracene has provided a useful intermediate for synthesis of other complicated arylvinylanthracenes.

\subsection{Solution and solid-state photophysical properties}

It is known that 1,4,5,8-positions of anthracene ring have strong repulsion on the aryl and vinyl groups linked at its 9- or 10-positions, which could result in strongly twisted backbone conformations. This should prevent from tight intermolecular stacking and usually render molecular backbone with intramolecular torsion motion that facilitates non-irradiation relaxation pathways. Like most other 9,10-bis(arylvinyl)anthracenes, SB and SN are also weakly emissive in THF solution with fluorescence quantum yields of $8.5 \%$ and $7.0 \%$, respectively. This could be explained in terms of freer intramolecular torsion motion in molecular solution. However, the THF solutions of ASB and ASN are highly fluorescent with fluorescence quantum yields up to $58.9 \%$ and $45.4 \%$, respectively. This implies that molecular backbone symmetry could affect significantly their emission behavior, although they all have intramolecular torsion motion moieties. Fig. 1 shows the absorption and emission spectra of the simple anthracene-based luminogens in THF solution. It is observed that absorption and emission of 9,10-bis(arylvinyl)anthracenes (SB and SN) are obviously red-shifted than those of 9-arylvinyl-anthracenes (ASB and ASN), which could be ascribed to the extension of conjugation size. On the other hand, naphthylvinyl- and phenylvinyl-anthracenes show the same absorption spectra, implying that the 1-position linking of naphthalene hardly affect the molecular conjugation degree. However, ASN (496 nm) and SN (599 $\mathrm{nm}$ ) exhibit red-shifted emission spectra than ASB (466 nm) and SB (567 nm), respectively. This implies that the existence of larger amplitude relaxation in 
the excited state for naphthyl than phenyl dyes, which is consistent with their fluorescence quantum yields.

All these simple anthracene-based luminogens are crystalline compounds, and their emission spectra in pristine crystalline states are shown in Fig. 2a. As observed above in solution, the crystalline emission spectra of symmetric compounds SB and SN (500 $\mathrm{nm}$ and $532 \mathrm{~nm}$ ) are red-shifter than those of mono-substituted ASB and ASN (460 nm and $470 \mathrm{~nm}$ ), and naphthylvinyl dyes (ASN and SN) exhibit bathochromic emission over phenylvinyl ones (ASB and SB). However, these crystalline emissions are obviously blue-shifted in comparison with the respective emissions in solution (Fig. 1). This phenomenon has been observed in other 9,10-bis(arylvinyl)anthracene derivatives and could be ascribed to the more twisted conformations in crystalline states for stabilizing crystal structure by forming strong supramolecular interactions. [19] It is noted that these crystals are all highly fluorescent, and their fluorescence efficiencies are measured to be $36.3 \%$ (ASB), 49.8\% (ASN), 44.1\% (SB), and 36.7\% (SN), respectively. Moreover, their evaporated films obtained by vacuum deposition are also highly fluorescent, and the fluorescence efficiencies are 33.5\% (ASB), 29.2\% (ASN), $25.8 \%$ (SB), and $22.4 \%(\mathbf{S N})$, respectively. This indicates that $\mathbf{S B}$ and $\mathbf{S N}$ are aggregation-enhanced emission dyes, and ASB and ASN are highly fluorescent dyes in both solution and solid-state. Fig. $2 b$ shows the emission spectra of evaporated ASB and ASN films. The evaporated films of ASB and ASN are blue and bluish-green emission with the peak wavelengths of $470 \mathrm{~nm}$ and 492 $\mathrm{nm}$, respectively, and their fluorescence colors and film morphologies maintain unchanged in the process of experimental operation. Contrarily, the evaporated SB and SN films re-crystallize and crack soon after the measurement on fluorescence efficiency, so that the emission spectra could not be recorded. Although the origin for these dichotomies is still unclear at present, this work has demonstrated that the subtle manipulation of backbone symmetry is an available way for filling the gap between aggregation-caused emission and aggregation-induced emission.

\subsection{Manchano-stimuli response behavior}

Conjugated organic materials exhibiting reversible solid-state fluorescence changes by external force stimuli instead of chemical alteration of the molecular structures have attracted immense interest due to their potential applications. Among them, 9,10-bis(arylvinyl)anthracene derivatives are a important class of mechanofluorochromic (MFC) dyes, but a recent report indicated that simple 9,10-distyrylanthracene (SB) has hardly MFC behavior by grinding in a mortar with a pestle [29]. Here we have investigated the MFC behavior of as-synthesized several simple anthracene-based derivatives, including SB, by grinding crystal powder on the glass plate with a metal spatula, and their fluorescence images under different external stimuli are 
shown in Fig. 3. It is found that the fluorescence colors of $\mathbf{S B}$, especially $\mathbf{S N}$, are obviously changed upon grinding and solvent-fuming over dichloromethane (2 min, at room temperature). Moreover, re-grinding could change again fluorescence color, and the changed color could recover upon annealing (heating $3 \mathrm{~min}$ in oven at $200{ }^{\circ} \mathrm{C}$ ). However, that is not the case for ASB and ASN, their fluorescence colors hardly change upon external stimuli, indicating that molecular backbone symmetry could affect their morphological stability and stimuli response behavior.

The fluorescence color changes above were recorded, and the emission spectra are depicted in Fig. 4. If denoting the peak-wavelength difference between ground and annealed states as the grinding-induced spectral shift $(\Delta \lambda)$, the $\Delta \lambda$ values of $\mathbf{S B}, \mathbf{S N}$, ASB, and ASN are 21, 23, 5, and $8 \mathrm{~nm}$, respectively. Bis-substitution dyes show more remarkable MFC behavior than mono- substitution ones. Fig. 5a is the differential scanning calorimetric curves of pristine crystals and ground solids, which shows that their melt points increase with molecular backbone size, but there is no notable information on aggregation morphological changes induced by grinding. In order to better understand those PFC behaviour, we also have carried out powder wide-angle X-ray diffraction (PWXD) measurements on the pristine and ground solids. PWXD measurements reveal these compounds exhibit different structures of molecular aggregation before and after grinding treatment (Fig. 5b). The diffraction patterns of the pristine samples display sharp and intense reflections, indicative of some crystalline order (Fig. 5b). However, the diffraction patterns of the ground samples show broaden and diffuse peaks, indicating destruction of pristine ordered structures, formation of crystal defects, and appearance of some content amorphous states. The existence of residual crystal seeds in the ground states is probably the reason that the ground states could change rapidly back to pristine states upon annealing or solvent-fuming.

\subsection{Electroluminescence}

Considering that commendable solid-state fluorescence efficiency and good morphological stability of ASB and ASN, their electroluminescence (EL) performances were investigated. The EL device with the configuration of ITO/ PEDOT:PSS (40 nm)/NPB (40 nm)/EML $(20 \mathrm{~nm}) / \mathrm{TPBi}(50 \mathrm{~nm}) / \mathrm{LiF}(0.5$ $\mathrm{nm}) / \mathrm{Al}(100 \mathrm{~nm})$ using ASB and ASN as emissive material layer (EML) was fabricated. As illustrated in Figure 6, the devices emit blue light at $479 \mathrm{~nm}$ and green light at $507 \mathrm{~nm}$ when ASB and ASN are used as EML, respectively. Their turn-on voltages are about $3.3 \sim 3.4 \mathrm{~V}$, a relative low value. Fig. 7 shows the Current density-luminance-voltage and the efficiency-voltage characteristics. The device with ASB as EML exhibits maximum luminance, current and power 
efficiencies of $3290 \mathrm{~cd} / \mathrm{m}^{2}, 1.68 \mathrm{~cd} / \mathrm{A}, 1.32 \mathrm{~lm} / \mathrm{W}$, respectively, and that with ASN as EML affords the improved EL performance with maximum luminance, current and power efficiencies of $5340 \mathrm{~cd} / \mathrm{m}^{2}, 3.67 \mathrm{~cd} / \mathrm{A}$ and $2.68 \mathrm{~lm} / \mathrm{W}$, respectively. These results are fairly commendable because of the simple structures of ASB and ASN without obvious electron- and hole-transporting moieties, and it already demonstrates the potential applications of monosubstitution 9-arylvinylanthracene derivatives in organic light-emitting diodes. To get further information on light-emitting material ASB and ASN, their electrochemical behaviors are investigated and the corresponding cyclic voltammograms are shown in Fig. 8a. The reversible oxidative and reductive behaviors are observed and indicate that they have the capability of hole- and electron-transporting. The oxidative and reductive potentials are measured as $\mathrm{E}_{\mathrm{ox}}=0.74$ and $0.73 \mathrm{eV}$ and $\mathrm{E}_{\mathrm{red}}=-2.07$ and $-2.02 \mathrm{eV}$ for ASB and ASN, respectively, implying that they have smaller difference in p-doping than $\mathrm{n}$-doping. Based on the onset oxidative and reductive potentials, their frontier molecular orbital energy levels are estimated to be -5.40 and $-5.40 \mathrm{eV}$ (HOMO) and -2.59, and -2.65 eV (LUMO) for ASB and ASN, respectively (Fig. 8b). The corresponding band gaps calculated are 2.81 and $2.75 \mathrm{eV}$, respectively, which are qualitatively consistent with the respective EL spectra (Fig. 6). It should be noted that, since the HOMO energy level of hole-transporting layer NPB is $-5.4 \mathrm{eV}$ and the LUMO energy level of electron-transporting layer TPBi is $-2.70 \mathrm{eV}$, hole injection from NPB, especially electron injection from TPBi to light-emitting layer is not very easy. This implies that the incorporation of both hole-transporting and electron- transporting units into the 9-arylvinylanthracenes should improve the EL performances.

\section{Conclusions}

We have designed and synthesized four simple arylvinyl-anthracene derivatives, ASB, ASN, SB, and SN to investigate the symmetry effect of conjugation backbone on the optical and optoelectronic properties. It is found that bis- substitution SB and $\mathbf{S N}$ are aggregation-enhanced emission dyes, and mono- substitution ASB and ASN emit intensely in both the molecularly dissolved solution and the solid states. Moreover, conjugation backbone symmetry could affect the stimuli response behavior and the morphological stability. SB and SN exhibit more obvious mechanofluorochromic than ASB and ASN, but the evaporated films are morphologically unstable and could re-crystallize soon. ASB and ASN evaporated films exhibit good morphological stability and high fluorescence efficiency could be used as light-emitting layer to afford commendable EL performances. These findings suggest that changing the molecular substitution pattern is an available way for filling the gap between aggregation-caused quenching and aggregation-induced emission, and subtle manipulation of conjugation backbone and aryl group could significantly 
alter and tune the optoelectronic properties. What's more, the asymmetry effect of conjugation backbone on the optical and optoelectronic properties will be investigated in next work.

\section{Acknowledgements}

We are grateful for the financial support from the NSF of China (51173092, 51303091), the NSF of Qingdao City (13-1-4-207-jch) and the Specialized Research Fund for the Doctoral Program of Higher Education (no. 201333 719120005). We also thank the State Key Laboratories of Luminescent Materials and Devices of South China University of Technology (SKLLMD 201408).

\section{References}

[1] G. Wang, K. Pu, X. Zhang, K. Li, L. Wang, L. Cai, D. Ding, Y. Lai, B. Liu, Chem. Mater. 23 (2011) 4428-4434.

[2] P. Liu, S. Li, Y.C. Jin, L.H. Qian, N.Y. Gao, S.Q. Yao, F. Huang, Q.H. Xu, Y. Gao, Appl. Mater. Interfaces. 7 (2015) 6754-6763.

[3] M. Sarma, T. Chatterjee, S. Ghanta, S.K. Das, J. Org. Chem. 77 (2012) 432-444.

[4] H.G. Lu, B. Xu, Y.J. Dong, F.P. Chen, Y.W. Li, Z.F. Li, J.T. He, H. Li, W.J Tian, Langmuir. 26 (2010) 6838-6844.

[5] Z.B. Jiang, Z.M. Zhong, S.F. Xue, Y. Zhou, Y.H. Meng, Z.H. Hu, N. Ai, J.B. Wang, L. Wang, J.B. Peng, Y.G Ma, J. Pei, J. Wang, Y. Gao, Appl. Mater. Interfaces. 6 (2014) 8345-8352.

[6] X.D. Wang, H. Li, Y.S. Wu, Z.Z. Xu, H.B. Fu, J. Am. Chem. Soc. 47 ( 2014) 16602-16608.

[7] K. Aggarwal, J. M. Khurana, J. Lumin. 167( 2015) 146-155

[8] Y.C. Li, X.L. Li, X.Y. Cai, D.C. Chen, X. Liu, G.Z. Xie, Y. Cao, S.J. Su, J. Mater. Chem. C 3(2015)6986-6996

[9] L.J. Yang, J.W. Ye, L.F. Xu, X.Y, Yang, W.T. Gong, Y. Lin, G.L. Ning, RSC Adv. 12 (2012) 11529-11535. 
[10] B. Xu, H.H. Fang, Y.J. Dong, F.P. Chen, Q.D. Chen, H.B. Sun, W.J. Tian, New J. Chem. 34 (2010) 1838-1842.

[11] M. Wang, G.X. Zhang, D.Q. Zhang, D.B. Zhu, B.Z. Tang, J. Mater. Chem. 20 (2010) 1858-1867.

[12] S. Kumar, S. Patil, J. Phys. Chem. C 119 (2015) 19297-19304.

[13] C.X. Shi, Z.Q. Guo, Y.L. Yan, S.Q. Zhu, Y.S. Xie, Y.S. Zhao, W.H. Zhu, H. Tian, Appl. Mater. Interfaces. 5 (2013) 192-198

[14] J.W. Chen, C.C.W. Law, J.W.Y. Lam, Y.P. Dong, S.M.F. Lo, I.D. Williams, D.B. Zhu, B. Tang, Chem. Mater. 15 (2003) 1535-1546.

[15] J.W. Chen, B. Xu, X.Y. Ouyang, B.Z. Tang, Y. Cao, J. Phys. Chem. A 108 (2004) 7522-7526.

[16] R.R. Hu, N.L.C.Lung, B.Z. Tang, Chem. Soc. Rev. 43 (2014) 4494-4562.

[17] J. Wang, J. Mei, R.R. Hu, J.Z. Sun, A.J. Qing, B.Z. Tang, J. Am. Chem. Soc. 134 (2012) 9956-9966.

[18] J.T. He, B. Xu, F.P. Chen, H.J. Xia, K.P. Li, L. Ye, W.J. Tian, J. Phys. Chem. C 113 (2009) 9892-9899.

[19] J.B. Zhang, B, Xu, J.L. Chen, L.J. Wang, W.J. Tian, J. Phys. Chem. C 117 (2013) 23117-23125.

[20] Y.Q. Dong, J.W.Y. Lam, A.J. Qin, J.X. Sun, J.Z. Liu, Z. Li, J.Z. Sun, H.H.Y. Sung, I.D. Willians, B.Z. Tang, Chem. Commun. 31 (2007) 3255-3257.

[21] C.M. Yang, I.W. Lee, T.L. Chen, W.L. Chien, J.L. Hong, J. Mater. Chem. C 1 (2013) 2842-2850.

[22] L.J. Qian, B. Tong, J.B. Shen, J.B. Shi, J.G. Zhi, Y.Q. Dong, F. Yang, Y.P. Dong, J.W.Y. Lam, B.Z. Tang, J. Phys. Chem. B 113 (2009) 9098-9103.

[23] S. Kim, Q.D. Zheng, G.S. He, D.J. Bharali, H.E. Pudavar, A. Baev, P.N. Prasad, Adv. Funct. Mater. 16 (2006) 2317-2323.

[24] S. Kim, H. Huang, H.E. Pudvar, Y.P. Cui, P.N. Prasad, Chem. Mater. 19 (2007) 5650-5656. 
1 [25] S. Kim, T.Y. Ohulchanskyy, H.E. Pudavar, R.K. Pandey, P.N. Prasad, J. Am. 2 Chem. Soc. 129 (2007) 2669-2675.

3

4 5

6

7

8

9

10

11

12

13

14

[26] M. Zheng, M.X. Sun, Y.P. Li, J.F. Wang, L.Y. Bu, S.F. Xue, W.J. Yang, Dyes Pigm. 102 (2014) 29-34.

[27] L.Y. Bu, Y.P. Li, J.F. Wang, M.X. Sun, W. Liu, S.F. Xue, W.J. Yang, Dyes Pigm. 99 (2013) 833-838.

[28] Y.L. Wang, W. Liu, L.Y. Bu, J.F. Li, M. Zheng. D.T. Zhang, M.X. Sun, Y. Tao, S.F. Xue, W.J. Yang, J. Mater. Chem. C 1 (2013) 856-862.

[29] X.Q. Zhang, Z.G. Chi, X. Zhou, S.W. Liu, Y. Zhang, J.R. Xu, J. Phys. Chem. C 116 (2012) 23629-23638. 


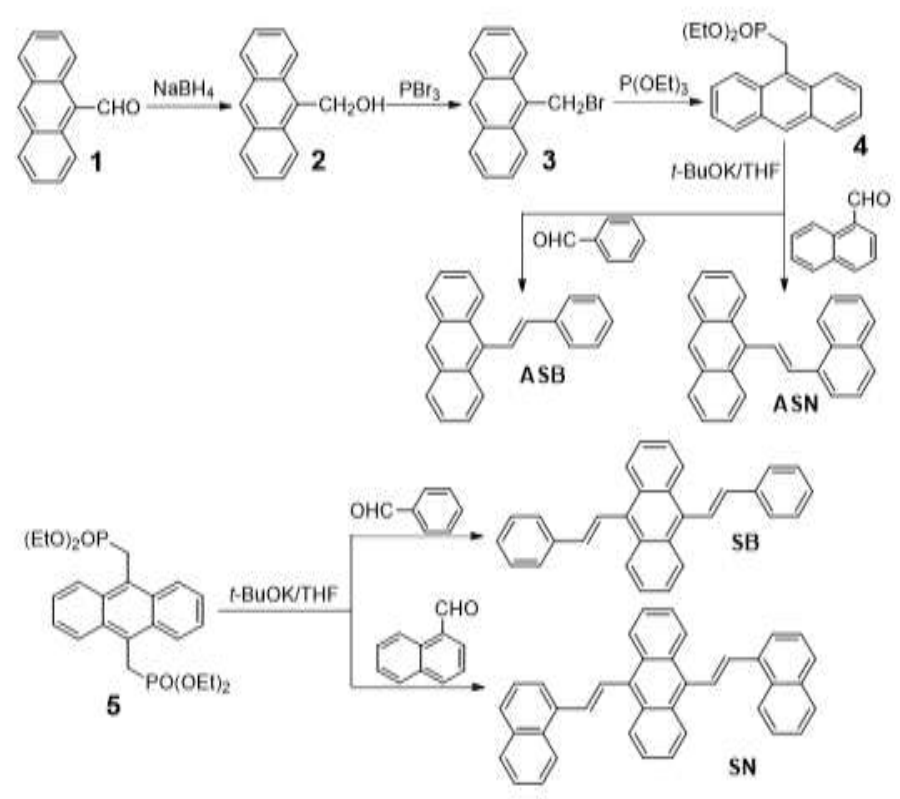

Scheme 1. Synthetic route and chemical structure of simple anthracene-based luminogens studied in this work. 

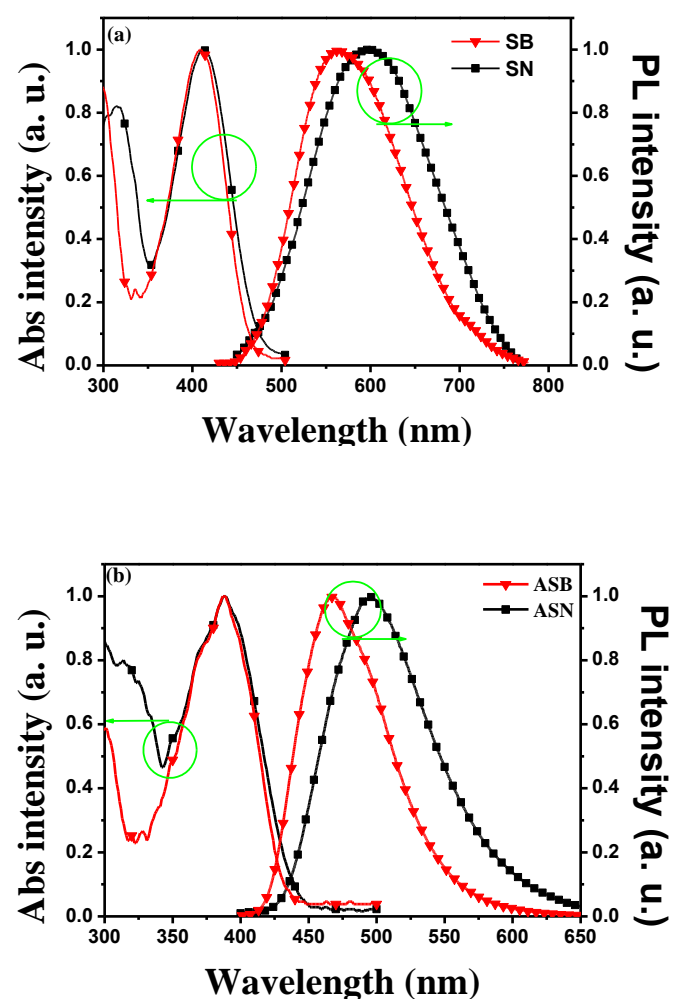

Fig. 1. Absorption and emission spectra of the simple anthracene-based luminogens in THF solution $\left(1.0 \times 10^{-5} \mathrm{M}\right)$. The peak wavelength of the lowest energy absorption band is used as the excitation wavelength(ASB/ASN,388nm). 

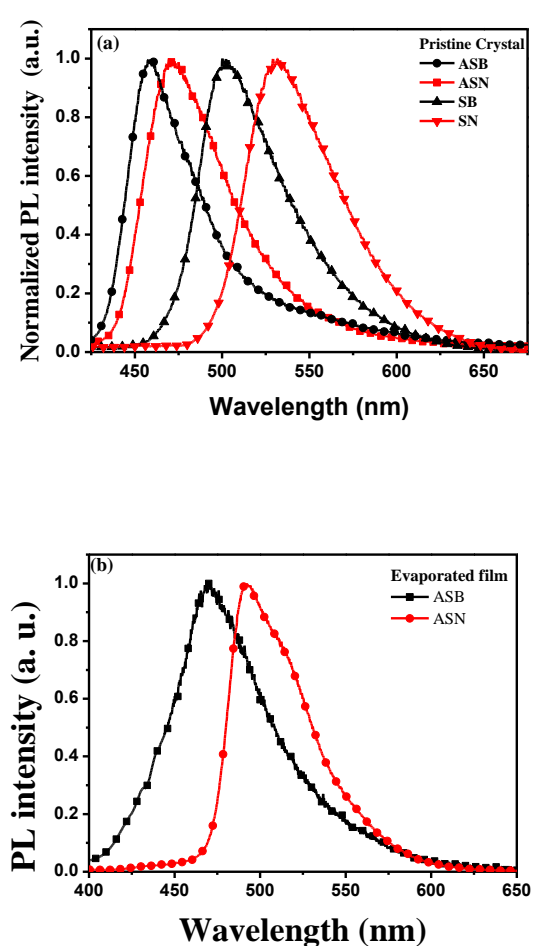

Fig. 2. Emission spectra of the simple anthracene-based luminogens under pristine crystal (a) and evaporated film (b) states. The excitation wavelength is at $400 \mathrm{~nm}$. 


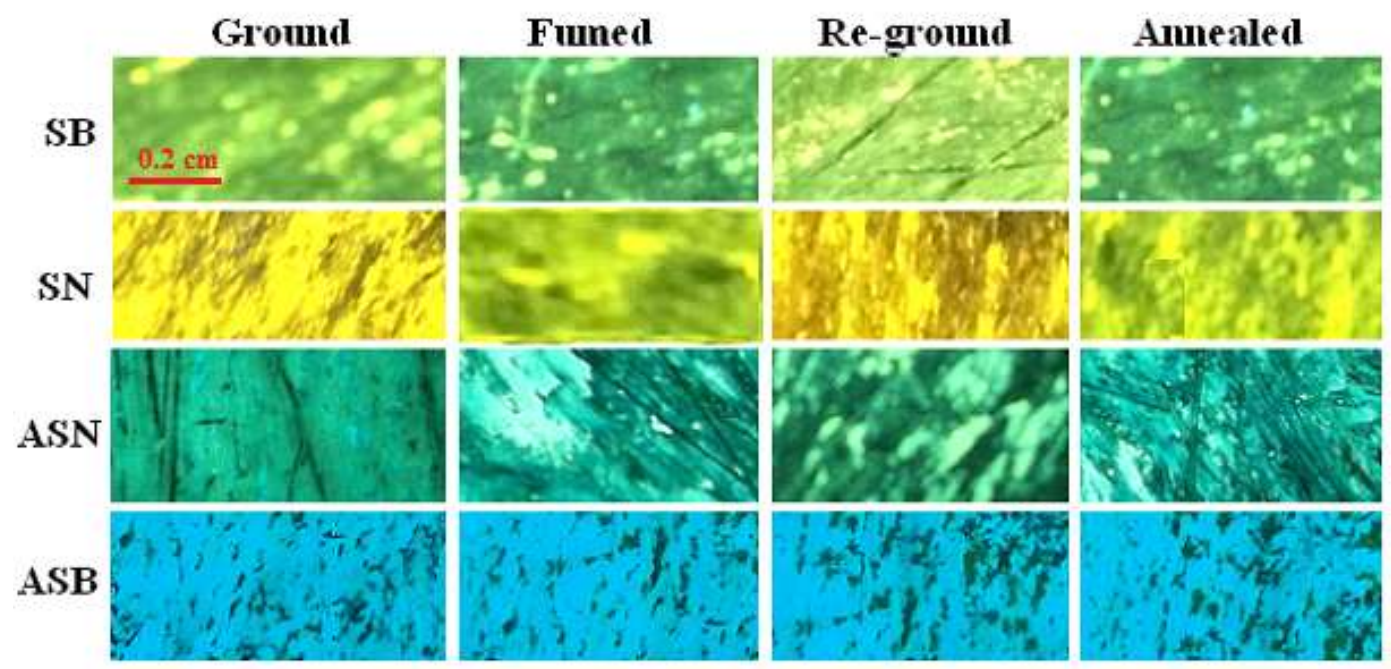

Fig. 3. Fluorescence images of the simple anthracene-based luminogens under different external stimuli with illumination of $365 \mathrm{~nm}$ UV lamp. 

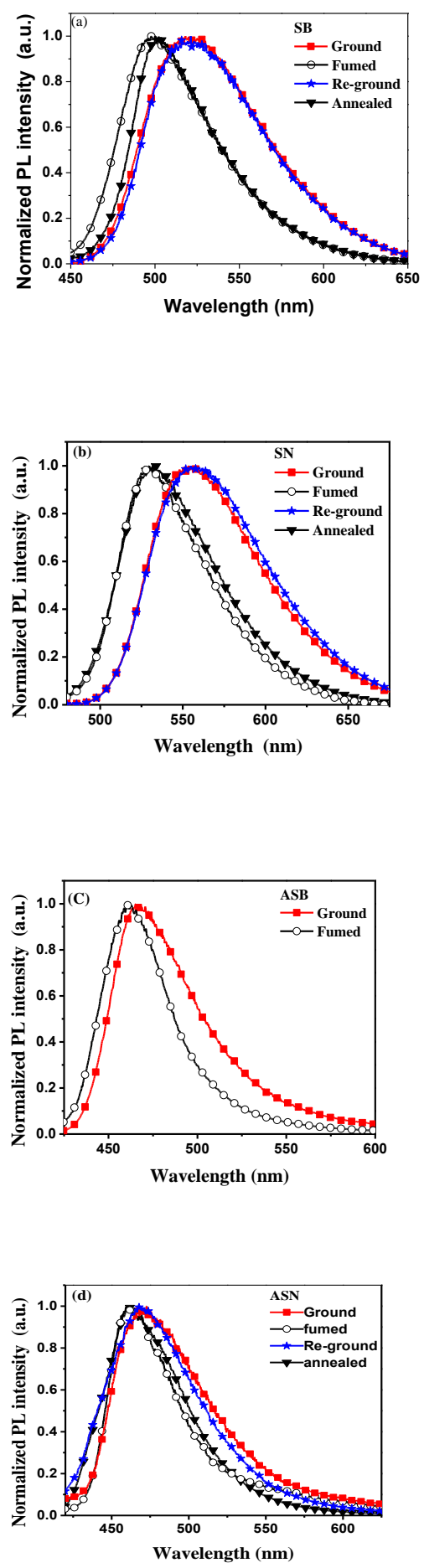

Fig. 4. Emission spectra of the simple anthracene-based luminogens under different external stimuli. The excitation wavelength is at $400 \mathrm{~nm}$. 

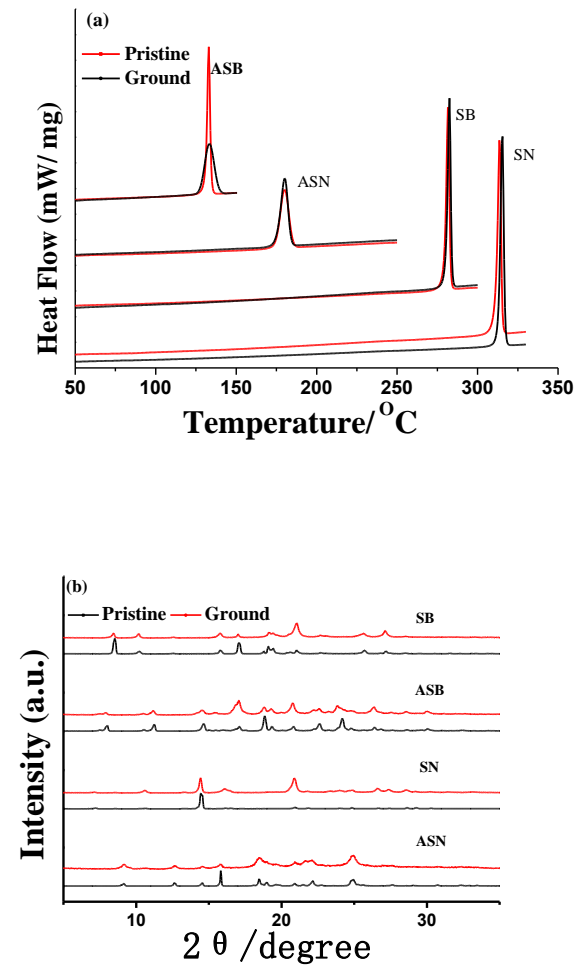

Fig. 5. (a) DSC curves of the simple anthracene-based luminogens under pristine and ground states. (b) Powder X-ray diffraction patterns at room temperature 


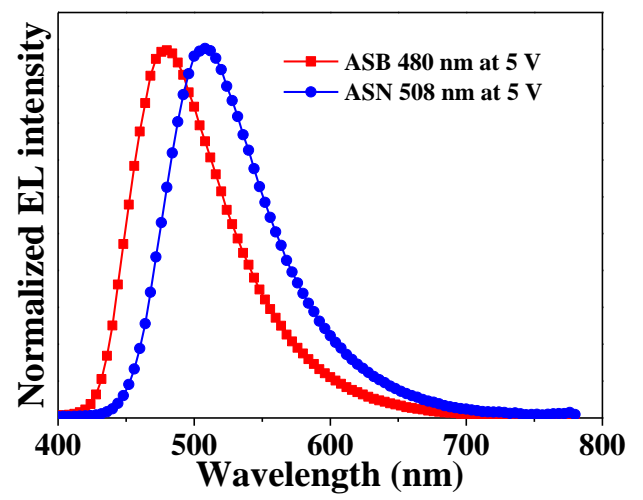

Fig. 6. Electroluminescence spectra of the light emitting diodes with ASB and ASN as the emitting layer. 

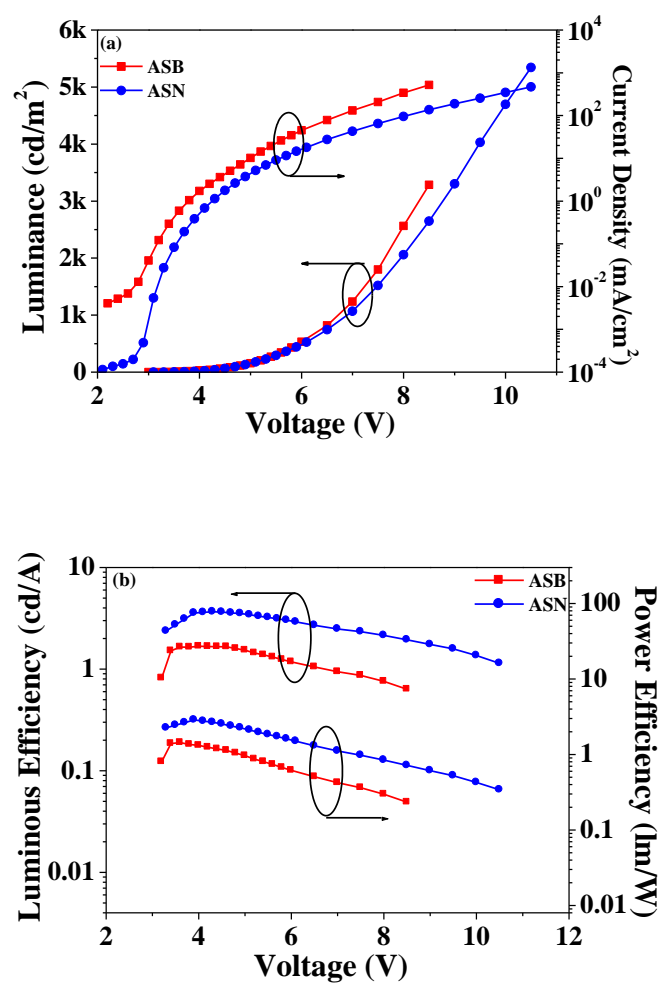

Fig. 7. (a) Current density-luminance-voltage and (b) the luminous efficiency-power efficiency-voltage characteristics of the ITO/PEDOT:PSS (40 nm)/NPB (40 $\mathrm{nm}) / \mathrm{EML}(20 \mathrm{~nm}) / \mathrm{TPBi}(50 \mathrm{~nm}) / \mathrm{LiF}(0.5 \mathrm{~nm}) / \mathrm{Al}(100 \mathrm{~nm})$. 

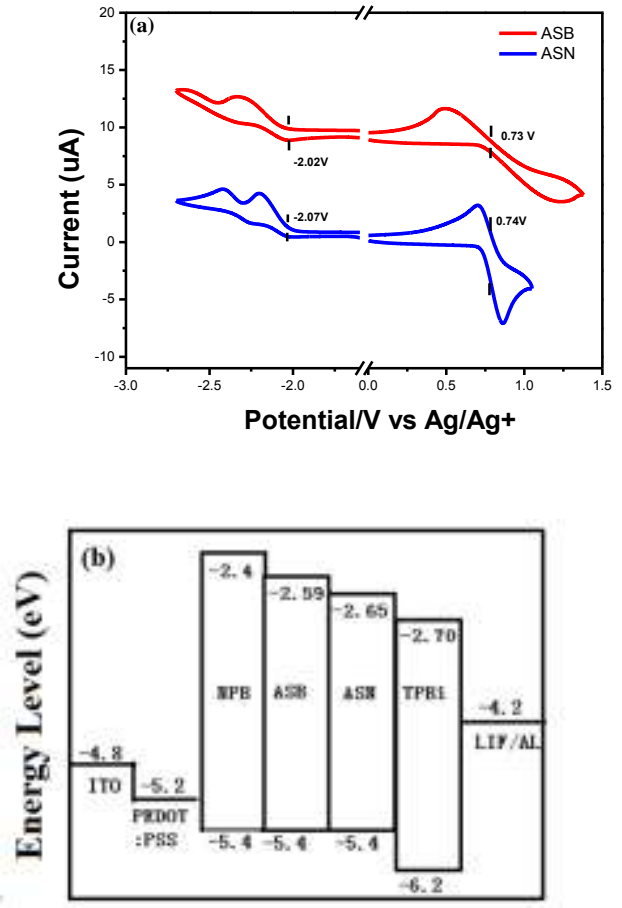

Fig. 8. (a) Cyclic voltammograms of ASB and $\mathbf{A S N}$ in $\mathrm{MeCN}$ with $0.1 \mathrm{M} \mathrm{Bu}_{4} \mathrm{NPF}_{6}$ as a supporting electrolyte at a scan rate of $100 \mathrm{mV} / \mathrm{s}$. (b) Energy diagram of the device with ASB and ASN as the emitting layer. 